PROCEEDINGS OF THE

AMERICAN MATHEMATICAL SOCIETY

Volume 137, Number 12, December 2009, Pages 4209-4218

S 0002-9939(09)10033-3

Article electronically published on July 31,2009

\title{
ON THE SOLVABILITY OF VECTOR FIELDS WITH REAL LINEAR COEFFICIENTS
}

\author{
FRANÇOIS TREVES
}

(Communicated by Bryna Kra)

\begin{abstract}
The following result is proved: for a vector field with real linear coefficients to be locally solvable in $\mathbb{R}^{n}$ it is necessary and sufficient that not all its orbits have a compact closure in the complement of the critical set of the vector field.
\end{abstract}

\section{INTRODUCTION}

In this paper the following notation is used: $\mathcal{C}_{\mathrm{c}}^{\infty}(\Omega)$ for the space of test functions in an open subset $\Omega$ of $\mathbb{R}^{n}$ and $\mathcal{D}^{\prime}(\Omega)$ for its dual, the space of distributions in $\Omega$. We write $\partial_{x_{j}}=\frac{\partial}{\partial x_{j}}(j=1, \ldots, n)$; the upper sign $\top$ indicates transpose (of a matrix or of an operator in a distribution space). By a linear change of variables we always mean a real linear change of variables.

We use the following terminology:

Definition 1. A smooth vector field $L$ in an open subset $\Omega$ of $\mathbb{R}^{n}$ is said to be locally solvable at a point $x^{\circ} \in \Omega$ if there exists an open neighborhood $U \subset \Omega$ of $x^{\circ}$ such that, to every $\varphi \in \mathcal{C}_{\mathrm{c}}^{\infty}(U)$, there is $u \in \mathcal{D}^{\prime}(\Omega)$ satisfying $L u=\varphi$ in $U$.

Our purpose is to characterize those vector fields in $\mathbb{R}^{n}$ whose coefficients are real linear functionals, i.e., vector fields of the form

$$
L=\sum_{j, k=1}^{n} a_{j k} x_{j} \partial_{x_{k}}
$$

that are locally solvable at every point of $\mathbb{R}^{n}$ (this will turn out to be equivalent to local solvability at the origin). The characterization is the content of Theorem 1 below; it extends to higher dimensions the two-dimensional result in Treves, 2009 (Theorem 10). Related results in the context of hyperfunctions can be found in Miwa, 1973 .

We denote by $\mathfrak{m}(L)$ the complex matrix $\left(a_{j k}\right)_{1 \leq j, k \leq n}$; then $\operatorname{div} L=\operatorname{tr} \mathfrak{m}(L)$, the trace of the matrix $\mathfrak{m}(L)$. Nonsingular linear changes of the variables $x_{1}, \ldots, x_{n}$ are equivalent to conjugations $\mathfrak{m}(L) \rightarrow \Gamma^{-1} \mathfrak{m}(L) \Gamma$ by a matrix $\Gamma \in \mathbf{G} L(n, \mathbb{R})$.

The critical points of $L$ are the points $x=\left(x_{1}, \ldots, x_{n}\right)$ in $\mathbb{R}^{n}$ such that $\mathfrak{m}(L)^{\top} \vec{x}=$ 0 . They form a vector subspace of $\mathbb{R}^{n}$, which will be denoted by $\boldsymbol{V}(L)$. We set

Received by the editors April 14, 2009, and, in revised form, April 29, 2009.

2000 Mathematics Subject Classification. Primary 35A07; Secondary 35F20.

Key words and phrases. Vector fields, local solvability, foliations.

(C)2009 American Mathematical Society 
$m=\operatorname{codim} \boldsymbol{V}(L)$; after a linear coordinate change we may assume that $\boldsymbol{V}(L)=$ $\left\{x \in \mathbb{R}^{n} ; x_{1}=\cdots=x_{m}=0\right\}$, in which case

$$
L=\sum_{j=1}^{m} \sum_{k=1}^{n} a_{j k} x_{j} \partial_{x_{k}} .
$$

The vector field (1.2) is invariant under translations in the variables $\left(x_{m+1}, \ldots, x_{n}\right)$, i.e., parallel to $\boldsymbol{V}(L)$; we have

$$
L=\sum_{j=1}^{m} x_{j} L_{j}, L_{j}=\sum_{k=1}^{n} a_{j k} \partial_{x_{k}} .
$$

Observe that the constant coefficients vector fields $L_{1}, \ldots, L_{m}$ must be linearly independent; if they were not, we would have $\operatorname{codim} \boldsymbol{V}(L)<m$.

When $L$ has the expression (1.2) we can identify $\mathbb{R}^{n} / \boldsymbol{V}(L)$ to $\mathbb{R}^{m}$ and the pushforward of $L$ under the quotient map $\mathbb{R}^{n} \longrightarrow \mathbb{R}^{n} / \boldsymbol{V}(L)$ to the vector field in $\mathbb{R}^{m}$,

$$
L^{\circ}=\sum_{j, k=1}^{m} a_{j k} x_{j} \frac{\partial}{\partial x_{k}} .
$$

We point out that the spectrum of $\mathfrak{m}(L)$ is equal to that of $\mathfrak{m}\left(L^{\circ}\right)$ to which the eigenvalue zero has been adjoined, with the multiplicity $n-m$; in particular, $\operatorname{div} L=$ $\operatorname{div} L^{\circ}$.

We note that $L$ defines a foliation of $\mathbb{R}^{n}$ whose leaves are either single points, precisely the critical points of $L$ [i.e., the points of $\boldsymbol{V}(L)$ ] or "true" curves contained in $\mathbb{R}^{n} \backslash \boldsymbol{V}(L)$. More precisely, the latter are immersed, connected, one-dimensional analytic submanifolds without self-intersections whose tangent line is spanned by $L$ at each one of their points (and are maximal for these properties). We shall refer to the leaves of the foliation defined by $L$ as $L$-orbits.

We shall avail ourselves of the following:

Lemma 1. The following properties of the vector field (1.2) are equivalent:

(1) every L-orbit in $\mathbb{R}^{n} \backslash \boldsymbol{V}(L)$ has a compact closure in $\mathbb{R}^{n} \backslash \boldsymbol{V}(L)$;

(2) every eigenvalue of $\mathfrak{m}\left(L^{\circ}\right)$ is purely imaginary, and the nilpotent part of $\mathfrak{m}\left(L^{\circ}\right)$ vanishes.

Proof. Let $\chi=\xi+i \eta(\xi, \eta \in \mathbb{R})$ be an eigenvalue of $\mathfrak{m}\left(L^{\circ}\right)$. If $\eta=0$ there is a linear change of the variables $x_{1}, \ldots, x_{m}$ such that $a_{11}=\xi$ and $a_{1 k}=0$ if $k=2, \ldots . m$. In the new coordinates,

$$
L=x_{1}\left(\xi \frac{\partial}{\partial x_{1}}+\sum_{k=m+1}^{n} a_{1 k} \frac{\partial}{\partial x_{k}}\right)+\sum_{j=2}^{m} \sum_{k=1}^{n} a_{j k} x_{j} \frac{\partial}{\partial x_{k}} .
$$

The vector field (1.5) is tangent to the linear subspace $x_{2}=\cdots=x_{m}=0$. In the half-subspaces $x_{1} \lessgtr 0$ the $L$-orbits coincide with those of the vector field

$$
\xi \frac{\partial}{\partial x_{1}}+\sum_{k=m+1}^{n} a_{1 k} \frac{\partial}{\partial x_{k}},
$$

which are obviously not compact. This contradicts (1).

Now suppose $\xi \eta \neq 0$ and let $\vec{u}=\vec{v}+i \vec{w} \in \mathbb{C}^{n}$ be such that $\mathfrak{m}\left(L^{\circ}\right) \vec{u}=(\xi+i \eta) \vec{u}$. We get

$$
\mathfrak{m}\left(L^{\circ}\right) \vec{v}=\xi \vec{v}-\eta \vec{w}, \mathfrak{m}\left(L^{\circ}\right) \vec{w}=\eta \vec{v}+\xi \vec{w} .
$$


Thus the span of $\vec{v}, \vec{w}$ is preserved by $\mathfrak{m}\left(L^{\circ}\right)$. We have necessarily $\vec{v} \wedge \vec{w} \neq 0$; otherwise $\mathfrak{m}\left(L^{\circ}\right)$ would have a real eigenvalue, a case already taken care of. A linear transformation enables us to assume that $\vec{v}=(1,0, \ldots, 0)$ and $\vec{w}=(0,1,0, \ldots, 0)$ and

$$
\mathfrak{m}\left(L^{\circ}\right)=\left(\begin{array}{ccc}
\xi & -\eta & 0 \\
\eta & \xi & 0 \\
* & * & *
\end{array}\right)
$$

The change of variables

$$
\tilde{x}_{j}=x_{j}-\frac{1}{\xi^{2}+\eta^{2}}\left(\left(\xi a_{1 j}+\eta a_{2 j}\right) x_{1}+\left(-\eta a_{1 j}+\xi a_{2 j}\right) x_{2}\right), j=m+1, \ldots, n,
$$

transforms

$$
\begin{aligned}
L_{1} & =\xi \partial_{x_{1}}-\eta \partial_{x_{2}}+\sum_{k=m+1}^{n} a_{1 k} \partial_{x_{k}}, \\
L_{2} & =\eta \partial_{x_{1}}+\xi \partial_{x_{2}}+\sum_{k=m+1}^{n} a_{2 k} \partial_{x_{k}}
\end{aligned}
$$

into

$$
L_{1}=\xi \partial_{x_{1}}-\eta \partial_{x_{2}}, L_{2}=\eta \partial_{x_{1}}+\xi \partial_{x_{2}} .
$$

As a result of this and of (1.3) we have (after deleting the tildes)

$$
L=x_{1}\left(\xi \partial_{x_{1}}-\eta \partial_{x_{2}}\right)+x_{2}\left(\eta \partial_{x_{1}}+\xi \partial_{x_{2}}\right)+\sum_{j=3}^{m} x_{j} L_{j} .
$$

When $L$ has the expression (1.6) $L$ is tangent to the deleted affine subspace $\dot{\boldsymbol{V}}$ defined by $x_{1}^{2}+x_{2}^{2} \neq 0, x_{3}=\cdots=x_{m}=0$. If we use polar coordinates in this subspace we see that

$$
\left.L\right|_{\dot{V}}=\xi r \partial_{r}-\eta \partial_{\theta}
$$

The orbits of $L$ in $\dot{\boldsymbol{V}}$ being the spirals $r=r^{\circ} \exp \left(-\eta^{-1} \xi \theta\right)$, no orbit of $L$ in $\dot{\boldsymbol{V}}$ has a compact closure, again contradicting (1).

Now suppose that $\xi=0, \eta \neq 0$, but that the nilpotent part of $\mathfrak{m}\left(L^{\circ}\right)$ does not vanish. If $i \eta(0 \neq \eta \in \mathbb{R})$ is an eigenvalue of $\mathfrak{m}\left(L^{\circ}\right)$, the same is true of $-i \eta$. There is a linear change of the variables $x_{1}, \ldots, x_{m}$ which puts $\mathfrak{m}\left(L^{\circ}\right)$ into the form

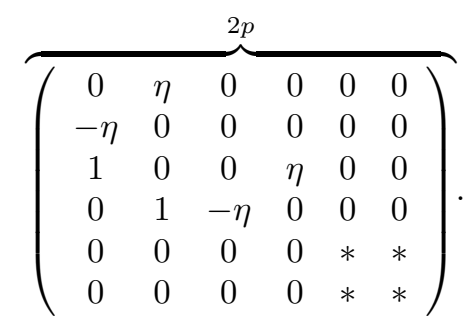

The expression of $L$ in the new coordinates will be

$$
\begin{aligned}
L= & \eta\left(x_{1} \partial_{x_{2}}-x_{2} \partial_{x_{1}}+x_{3} \partial_{x_{4}}-x_{4} \partial_{x_{3}}\right) \\
& +x_{3} \partial_{x_{1}}+x_{4} \partial_{x_{2}}+\sum_{j=5}^{m} \sum_{k=m+1}^{n} a_{j k} x_{j} \partial_{x_{k}} .
\end{aligned}
$$


When $L$ has the expression (1.7), $L$ is tangent to the deleted affine subspace $\dot{\boldsymbol{V}}$ defined by $x_{1}^{2}+x_{2}^{2}+x_{3}^{2}+x_{4}^{2} \neq 0, x_{5}=\cdots=x_{n}=0$. We see that

$$
\left.L\right|_{\dot{\boldsymbol{V}}}=\eta\left(x_{1} \partial_{x_{2}}-x_{2} \partial_{x_{1}}+x_{3} \partial_{x_{4}}-x_{4} \partial_{x_{3}}\right)+x_{3} \partial_{x_{1}}+x_{4} \partial_{x_{2}}
$$

It is checked straightaway that $L$ is tangent to the curve in $\dot{\boldsymbol{V}}$ defined by the parametric equations

$$
x_{1}=\eta^{-1} t \cos t, x_{2}=\eta^{-1} t \sin t, x_{3}=\cos t, x_{4}=\sin t, t \in \mathbb{R},
$$

whose closure in $\dot{\boldsymbol{V}}$ is noncompact, contradicting (1). We have thus proved that the negation of (2) implies that of (1).

Now suppose $(2)$ is true. There is a linear change of the variables $x_{1}, \ldots, x_{m}$ that puts $L^{\circ}$ into the form

$$
L^{\circ}=\sum_{j=1}^{p} \eta_{j}\left(x_{2 j-1} \partial_{x_{2 j}}-x_{2 j} \partial_{x_{2 j-1}}\right),
$$

and therefore $L$ into the form

$$
L=\sum_{j=1}^{p} \eta_{j}\left(x_{2 j-1} L_{2 j}-x_{2 j} L_{2 j-1}\right),
$$

where

$$
L_{k}=\partial_{x_{k}}+\sum_{\ell=2 p+1}^{n} b_{k \ell} \partial_{\ell}, k=1, \ldots, 2 p .
$$

We carry out the change of variables

$$
\begin{aligned}
& \tilde{x}_{k}=x_{k}, k=1, \ldots, 2 p, \\
& \tilde{x}_{\ell}=x_{\ell}-\sum_{k=1}^{2 p} b_{k \ell} x_{k}, \ell=2 p+1, \ldots, n .
\end{aligned}
$$

In the new coordinates, $L_{k}=\partial_{\tilde{x}_{k}}$ which, after deletion of the tildes, puts $L$ itself into the form (1.8). If we write $x_{2 j-1}=r_{j} \cos \theta_{j}, x_{2 j}=r_{j} \sin \theta_{j}$, we get

$$
L=\sum_{j=1}^{p} \eta_{j} \partial_{\theta_{j}}
$$

We see that the $L$-orbit through a point $x^{\circ} \in \mathbb{R}^{n} \backslash \boldsymbol{V}(L)$ is a geodesic of the torus

$$
\begin{array}{r}
\widetilde{\mathbb{T}}^{2 p}\left(x^{\circ}\right)=\left\{x \in \mathbb{R}^{n} \backslash \boldsymbol{V}(L) ; x_{j}^{2}+x_{p+j}^{2}=\left(x_{j}^{\circ}\right)^{2}+\left(x_{p+j}^{\circ}\right)^{2}, j=1, \ldots, p,\right. \\
\left.x_{k}=x_{k}^{\circ}, k=2 p+1, \ldots, n\right\} .
\end{array}
$$

As a consequence, the closure of every $L$-orbit is compact.

Corollary 1. If $\operatorname{div} L \neq 0$, then there is an L-orbit in $\mathbb{R}^{n} \backslash \boldsymbol{V}(L)$ whose closure is not a compact subset of $\mathbb{R}^{n} \backslash \boldsymbol{V}(L)$.

Proof. If $\operatorname{tr} \mathfrak{m}(L) \neq 0$ there is an eigenvalue of $\mathfrak{m}(L)$ whose real part does not vanish.

Remark 1. Property (2) in Lemma 1 is equivalent to the following property:

- The one-parameter subgroup $\mathbb{R} \ni t \longrightarrow \exp t \mathfrak{m}(L) \in \mathbf{G} L(n, \mathbb{R})$ has a compact closure. 


\section{Statement AND PROOF OF THEOREM}

\subsection{Statement of theorem and first observations.}

Theorem 1. The following properties of the vector field (1.1) are equivalent:

(a) $L$ is not locally solvable at any point of $\boldsymbol{V}(L)$.

(b) The closure of every L-orbit in $\mathbb{R}^{n} \backslash \boldsymbol{V}(L)$ is a compact subset of $\mathbb{R}^{n} \backslash \boldsymbol{V}(L)$.

(c) There are coordinates $x_{1}, \ldots, x_{n}$ in $\mathbb{R}^{n}$ such that the L-orbit of an arbitrary point $x^{\circ} \in \mathbb{R}^{n} \backslash \boldsymbol{V}(L)$ is a geodesic of the torus (1.10).

Remark 2. Owing to the invariance of $L$ under translations in the $\boldsymbol{V}(L)$ directions, Property (a) could have as well been stated as

$\left(\mathbf{a}^{\prime}\right) L$ is not locally solvable at the origin.

Remark 3. Property (c) requires $m=\operatorname{dim} \mathbb{R}^{n} / \boldsymbol{V}(L)=2 p\left(0 \neq p \in \mathbb{Z}_{+}\right)$. Lemma 1 states that (b) is equivalent to the property that the nonzero eigenvalues of $\mathfrak{m}(L)$ are purely imaginary numbers $\pm i \eta_{j}(j=1, \ldots, p)$ and the nilpotent part of $\mathfrak{m}\left(L^{\circ}\right)$ vanishes. The real numbers $\eta_{j}$ determine which geodesic in the torus (1.10) is the $L$-orbit through $x^{\circ}$.

That (c) implies (b) is trivial; the converse is part of Lemma 1, Proving that (a) and (b) are equivalent will be a matter of settling a few special cases. The corresponding results will be stated as independent propositions. The proofs of these propositions will make repeated use of the next two classical lemmas. In what follows $(\cdot, \cdot)$ and $\|\cdot\|$ stand for the inner product and the norm in $L^{2}(\Omega)$, respectively.

Lemma 2. If $X$ is a vector field with constant coefficients, not all vanishing, and if $h$ is a complex-valued analytic function in an open subset $\Omega$ of $\mathbb{R}^{n}$, then the vector field $h(x) X$ is locally solvable in $\Omega$.

Proof. An arbitrary distribution $f \in \mathcal{D}^{\prime}(\Omega)$ can be divided by the analytic function $h$ ([Lojasiewicz, 1965]). Then it suffices to solve the equation $X u=h^{-1} f$.

Lemma 3. Let $\Omega$ be an open subset of $\mathbb{R}^{n}$. Suppose there is a linear partial differential operator with constant coefficients $P(\partial)$ such that, for some $C>0$ and all $\varphi \in \mathcal{C}_{c}^{\infty}(\Omega)$,

$$
\|\varphi\| \leq C\left\|P(\partial) L^{\top} \varphi\right\|
$$

Then $L \mathcal{D}^{\prime}(\Omega) \supset L^{2}(\Omega)$.

Proof. The estimate (2.1) entails that the linear map $P(\partial) L^{\top} \mathcal{C}_{\mathrm{c}}^{\infty}(\Omega) \ni P(\partial) L^{\top} \varphi$ $\longrightarrow \varphi \in \mathcal{C}_{\mathrm{c}}^{\infty}(\Omega)$ extends as a bounded linear operator $E: L^{2}(\Omega) \hookleftarrow$ having the property that $E P(\partial) L^{\top} \varphi=\varphi$ for all $\varphi \in \mathcal{C}_{\mathrm{c}}^{\infty}(\Omega)$. We derive that $L P(-\partial) E^{\top} f=$ $f$ for every $f \in L^{2}(\Omega)$. tions.

We begin the proof proper of Theorem 1 with the following two simple observa-

The case $m=1$ is a direct consequence of Lemma 2 since, in this case, $L=x_{1} L_{1}$ with $L_{1}$ having constant coefficients not all vanishing. The $L$-orbits are the same as the orbits of $X$ (which are straight lines) possibly "interrupted" by the hyperplane $x_{1}=0$.

In the remainder of the proof we limit our attention to the cases $2 \leq m \leq n$. 
Proposition 1. Let $L$ be the vector field (1.2). If $\operatorname{div} L \neq 0$, then there is a bounded linear operator $E$ on $L^{2}\left(\mathbb{R}^{n}\right)$ such that $L E f=f$ for all $f \in L^{2}\left(\mathbb{R}^{n}\right)$.

Proof. We have, whatever $\varphi \in \mathcal{C}_{c}^{\infty}\left(\mathbb{R}^{n}\right)$,

$$
2 \operatorname{Re}\left(L^{\top} \varphi, \varphi\right)_{L^{2}}=-(\operatorname{div} L)\|\varphi\|_{L^{2}} .
$$

The claim follows then from the Cauchy-Schwarz inequality and Lemma 3.

In the sequel we restrict our attention to divergence-free vector fields (1.1), in which case $L^{\top}=-L$.

2.2. The matrix $\mathfrak{m}\left(L^{\circ}\right)$ has at least one real eigenvalue $\xi$. In this case we may assume that $L$ has the form (1.5).

Proposition 2. Let $L$ be the vector field (1.5). If $\operatorname{div} L=0$ and if $\mathfrak{m}\left(L^{\circ}\right)$ has at least one real eigenvalue $\xi$ (possibly equal to zero), then $L$ is locally solvable in $\mathbb{R}^{n}$.

Proof. We may assume that $L$ is the vector field (1.5).

Case $\xi \neq 0$. We use the fact that $\partial_{x_{1}} L=(L+\xi) \partial_{x_{1}}$. Since $\operatorname{div} L=0$ we have, for all $\varphi \in \mathcal{C}_{c}^{\infty}\left(\mathbb{R}^{n}\right)$,

$$
\operatorname{Re}((L+\xi) \varphi, \varphi)_{L^{2}}=\xi\|\varphi\|_{L^{2}},
$$

implying

$$
\operatorname{Re}\left(\partial_{x_{1}}^{2} L \varphi, \varphi\right)_{L^{2}}=\operatorname{Re}\left(\partial_{x_{1}}(L+\xi) \partial_{x_{1}} \varphi, \varphi\right)_{L^{2}}=-\xi\left\|\partial_{x_{1}} \varphi\right\|_{L^{2}} .
$$

To every number $T>0$ there is $C_{T}>0$ such that $\|\varphi\|_{L^{2}} \leq C_{T}\left\|\partial_{x_{1}} \varphi\right\|_{L^{2}}$ for every $\varphi$ whose support is contained in the slab $(-T, T) \times \mathbb{R}^{n-1}$. The claim then follows from the Cauchy-Schwarz inequality and Lemma 3.

Case $\xi=0$. In this case we have $L=x_{1} \sum_{k=m+1}^{n} a_{1 k} \frac{\partial}{\partial x_{k}}+\sum_{j=2}^{m} \sum_{k=1}^{n} a_{j k} x_{j} \frac{\partial}{\partial x_{k}}$ according to (1.5). There is a linear change of the variables $x_{m+1}, \ldots ., x_{n}$ that transforms $L$ into

$$
x_{1} \partial_{x_{m+1}}+\sum_{j=2}^{m} x_{j}\left(\sum_{1 \leq k \leq n} a_{j k} \partial_{x_{k}}\right) .
$$

Since $\operatorname{div} L=0$ we see that

$$
2 \operatorname{Re}\left(L \varphi, \partial_{x_{1}} \partial_{x_{m+1}} \varphi\right)_{L^{2}}=-\left\|\partial_{x_{m+1}} \varphi\right\|_{L^{2}}^{2}
$$

for every $\varphi \in \mathcal{C}_{\mathrm{c}}^{\infty}\left(\mathbb{R}^{n}\right)$. We define $\Omega_{T}=\left\{x \in \mathbb{R}^{n} ;\left|x_{m+1}\right|<T\right\}(T>0)$. There is $C_{T}>0$ such that

$$
\|\varphi\|_{L^{2}} \leq C_{T}\left\|\partial_{x_{m+1}} \varphi\right\|_{L^{2}}
$$

for all $\varphi \in \mathcal{C}_{\mathrm{c}}^{\infty}\left(\Omega_{T}\right)$. That the vector field (2.2) is locally solvable in $\mathbb{R}^{n}$ then ensues directly from the Cauchy-Schwarz inequality and Lemma 3 


\subsection{The matrix $\mathfrak{m}\left(L^{\circ}\right)$ has no real eigenvalues.}

2.3.1. Case I: The real part of some eigenvalue of $\mathfrak{m}\left(L^{\circ}\right)$ is different from zero.

Proposition 3. Let $L$ be the vector field (1.2). If $\operatorname{div} L=0$ and if $\mathfrak{m}\left(L^{\circ}\right)$ has at least one eigenvalue whose real part is different from zero, then $L$ is locally solvable in $\mathbb{R}^{n}$.

Proof. We may assume that $L$ has the expression (1.6); it follows that, for every $\varphi \in \mathcal{C}_{\mathrm{c}}^{\infty}\left(\mathbb{R}^{n}\right)$,

$$
\begin{aligned}
& \partial_{x_{1}} L \varphi=L\left(\partial_{x_{1}} \varphi\right)+\xi \partial_{x_{1}} \varphi-\eta \partial_{x_{2}} \varphi \\
& \partial_{x_{2}} L \varphi=L\left(\partial_{x_{2}} \varphi\right)+\eta \partial_{x_{1}} \varphi+\xi \partial_{x_{2}} \varphi
\end{aligned}
$$

whence

$$
\left(\partial_{x_{1}}+i \partial_{x_{2}}\right) L \varphi=L\left(\partial_{x_{1}} \varphi+i \partial_{x_{2}} \varphi\right)+(\xi+i \eta)\left(\partial_{x_{1}} \varphi+i \partial_{x_{2}} \varphi\right) .
$$

Set $\Phi=\partial_{x_{1}} \varphi+i \partial_{x_{2}} \varphi$; we have

$$
\left(\partial_{x_{1}}+i \partial_{x_{2}}\right) L \varphi=L \Phi+(\xi+i \eta) \Phi
$$

and

$$
\operatorname{Re}(L \Phi+(\xi+i \eta) \Phi, \Phi)_{L^{2}}=\xi\|\Phi\|_{L^{2}} .
$$

But this can be rewritten as

$$
\operatorname{Re}\left(\left(\partial_{x_{1}}+i \partial_{x_{2}}\right)^{2} L \varphi, \varphi\right)_{L^{2}}=\xi\left\|\partial_{x_{1}} \varphi+i \partial_{x_{2}} \varphi\right\|_{L^{2}} .
$$

Let $\Omega_{R}=\left\{x \in \mathbb{R}^{n} ; x_{1}^{2}+x_{2}^{2}<R\right\}, R>0$. We have, for some $C_{R}>0$ and all $\varphi \in \mathcal{C}_{\mathrm{c}}^{\infty}\left(\Omega_{R}\right)$,

$$
\|\varphi\|_{L^{2}} \leq C_{R}\left\|\partial_{x_{1}} \varphi+i \partial_{x_{2}} \varphi\right\|_{L^{2}} .
$$

The Cauchy-Schwarz inequality and Lemma 3 entail the claim.

2.3.2. Case II: Every eigenvalue of $\mathfrak{m}\left(L^{\circ}\right)$ is purely imaginary. We continue to assume that $\operatorname{div} L=0$.

Proposition 4. If all the eigenvalues of $\mathfrak{m}\left(L^{\circ}\right)$ are purely imaginary and if the nilpotent part of $\mathfrak{m}\left(L^{\circ}\right)$ is not equal to zero, then $L$ is locally solvable at every point of $\boldsymbol{V}(L)$.

Proof. In this case we may assume that $L$ has the expression (1.7). We derive

$$
\begin{gathered}
\partial_{x_{1}} L \varphi=L \partial_{x_{1}} \varphi+\eta \partial_{x_{2}} \varphi \\
\partial_{x_{2}} L \varphi=L \partial_{x_{2}} \varphi-\eta \partial_{x_{1}} \varphi \\
\partial_{x_{3}} L \varphi=L \partial_{x_{3}} \varphi+\eta \partial_{x_{4}} \varphi+\partial_{x_{1}} \varphi \\
\partial_{x_{4}} L \varphi=L \partial_{x_{4}} \varphi-\eta \partial_{x_{3}} \varphi+\partial_{x_{2}} \varphi .
\end{gathered}
$$

Integration by parts implies

$$
\begin{aligned}
\left(\partial_{x_{3}} L \varphi, \partial_{x_{1}} \varphi\right) & =\left(L \partial_{x_{3}} \varphi, \partial_{x_{1}} \varphi\right)+\eta\left(\partial_{x_{4}} \varphi, \partial_{x_{1}} \varphi\right)+\left\|\partial_{x_{1}} \varphi\right\|^{2} \\
& =-\left(\partial_{x_{3}} \varphi, L \partial_{x_{1}} \varphi\right)+\eta\left(\partial_{x_{4}} \varphi, \partial_{x_{1}} \varphi\right)+\left\|\partial_{x_{1}} \varphi\right\|^{2} \\
& =-\left(\partial_{x_{3}} \varphi, \partial_{x_{1}} L \varphi\right)+\eta\left(\partial_{x_{3}} \varphi, \partial_{x_{2}} \varphi\right)+\eta\left(\partial_{x_{4}} \varphi, \partial_{x_{1}} \varphi\right)+\left\|\partial_{x_{1}} \varphi\right\|^{2}
\end{aligned}
$$

whence

$$
2 \operatorname{Re}\left(\partial_{x_{3}} L \varphi, \partial_{x_{1}} \varphi\right)=\left\|\partial_{x_{1}} \varphi\right\|^{2}+\eta\left(\left(\partial_{x_{3}} \varphi, \partial_{x_{2}} \varphi\right)+\left(\partial_{x_{4}} \varphi, \partial_{x_{1}} \varphi\right)\right)
$$


Likewise,

$$
\begin{aligned}
\left(\partial_{x_{4}} L \varphi, \partial_{x_{2}} \varphi\right) & =\left(L \partial_{x_{4}} \varphi, \partial_{x_{2}} \varphi\right)-\eta\left(\partial_{x_{3}} \varphi, \partial_{x_{2}} \varphi\right)+\left\|\partial_{x_{2}} \varphi\right\|^{2} \\
& =-\left(\partial_{x_{4}} \varphi, L \partial_{x_{2}} \varphi\right)-\eta\left(\partial_{x_{3}} \varphi, \partial_{x_{2}} \varphi\right)+\left\|\partial_{x_{2}} \varphi\right\|^{2} \\
& =-\left(\partial_{x_{4}} \varphi, \partial_{x_{2}} L \varphi\right)-\eta\left(\partial_{x_{4}} \varphi, \partial_{x_{1}} \varphi\right)-\eta\left(\partial_{x_{3}} \varphi, \partial_{x_{2}} \varphi\right)+\left\|\partial_{x_{2}} \varphi\right\|^{2}
\end{aligned}
$$

whence

$$
2 \operatorname{Re}\left(\partial_{x_{4}} L \varphi, \partial_{x_{2}} \varphi\right)=\left\|\partial_{x_{2}} \varphi\right\|^{2}-\eta\left(\left(\partial_{x_{3}} \varphi, \partial_{x_{2}} \varphi\right)+\left(\partial_{x_{4}} \varphi, \partial_{x_{1}} \varphi\right)\right) .
$$

We conclude that

$$
2 \operatorname{Re}\left(\left(\partial_{x_{3}} L \varphi, \partial_{x_{1}} \varphi\right)+\left(\partial_{x_{4}} L \varphi, \partial_{x_{2}} \varphi\right)\right)=\left\|\partial_{x_{1}} \varphi\right\|^{2}+\left\|\partial_{x_{2}} \varphi\right\|^{2}
$$

for all $\varphi \in \mathcal{C}_{\mathrm{c}}^{\infty}\left(\mathbb{R}^{2}\right)$. In view of (2.3) the Cauchy-Schwarz inequality and Lemma 3 entail the claim.

The proof of Theorem 1 is completed with the following result.

Proposition 5. If all the eigenvalues of $\mathfrak{m}\left(L^{\circ}\right)$ are purely imaginary and the nilpotent part of $\mathfrak{m}\left(L^{\circ}\right)$ is equal to zero, then $L$ is not locally solvable at any point of $\boldsymbol{V}(L)$.

Proof. In this case we may assume that $L=\sum_{j=1}^{p} \eta_{j} \partial_{\theta_{j}}$ [see (1.9)], where $\theta_{j}=$ $\arg \left(x_{2 j-1}+\sqrt{-1} x_{2 j}\right)$. Let $\mathfrak{B}_{R}=\left\{x \in \mathbb{R}^{n} ; \sum_{j=1}^{n} x_{j}^{2}<R^{2}\right\}, R>0$. If $u \in$ $\mathcal{D}^{\prime}\left(\mathfrak{B}_{R}\right)$ and $F \in \mathcal{C}_{\mathrm{c}}^{\infty}\left(\mathfrak{B}_{R}\right)$ are such that $L u=F$ in $\mathfrak{B}_{R}$, then necessarily

$\int_{0}^{2 \pi} \cdots \int_{0}^{2 \pi} F\left(r_{1} \cos \theta_{1}, r_{1} \sin \theta_{1}, \ldots, r_{p} \cos \theta_{p}, r_{p} \sin \theta_{p}, \tilde{x}_{m+1}, \ldots, \tilde{x}_{n}\right) d \theta_{1} \cdots d \theta_{p}=0$

for all $r_{j} \geq 0$ and all $\left(\tilde{x}_{m+1}, \ldots, \tilde{x}_{n}\right) \in \mathbb{R}^{n-m}: F$ cannot be an arbitrary test function in $\mathfrak{B}_{R}$.

\section{VeCTOR FIELDS WiTH COMPLEX LINEAR COEFFICIENTS DEFINING A RANK-ONE FOLIATION}

In this section we allow the coefficients of the vector field (1.1) to be complex. We reason under the hypothesis that $L$ is locally solvable in the open set $\Omega=\mathbb{R}^{n} \backslash \boldsymbol{V}(L)$. We begin by recalling what this entails. Let $\mathfrak{g}(L)$ denote the Lie algebra generated by the real vector fields $X=\operatorname{Re} L, Y=\operatorname{Im} L$. The Nagano foliation of $\mathbb{R}^{n} \backslash \boldsymbol{V}(L)$ defined by $\mathfrak{g}(L)$ consists of connected analytic submanifolds whose tangent space at any one of their points is equal to the "freezing" of $\mathfrak{g}(L)$ at that point and which are maximal for these properties. A classical result of the local solvability theory of smooth complex vector fields without critical points (see Treves, 1992, Section VIII.7) states that for $L$ to be locally solvable in $\mathbb{R}^{n} \backslash \boldsymbol{V}(L)$ it is necessary and sufficient that the following requirement be satisfied:

(P) The leaves of the Nagano foliation of $\mathbb{R}^{n} \backslash \boldsymbol{V}(L)$ defined by the Lie algebra $\mathfrak{g}(L)$ have dimension 1 or 2 . On each two-dimensional leaf the sign of $\frac{1}{2 i} L \wedge \bar{L}$ does not change.

Here we shall assume that there are no two-dimensional Nagano leaves and we shall not concern ourselves with the sign of $\frac{1}{2 i} L \wedge \bar{L}$. To say that there are only onedimensional Nagano leaves is equivalent to saying that $L \wedge \bar{L}$ vanishes identically. The extension of Theorem 1 is not quite automatic since the vanishing of $L \wedge \bar{L}$ at 
every point does not imply that $\zeta^{-1} L$ is a real vector field for some $\zeta \in \mathbb{C} \backslash\{0\}$. We take a closer look at this question.

In the notation (1.3),$L \wedge \bar{L} \equiv 0$ is equivalent to the equations

$$
\operatorname{Im}\left(L_{j} \wedge \bar{L}_{k}\right)=0
$$

$(1 \leq j \leq k \leq m)$. For each $j=1, \ldots, m$, we can select a real vector field $L_{j}^{\mathbb{R}} \neq 0$ such that $L_{j}=\zeta_{j} L_{j}^{\mathbb{R}}$ for some $\zeta_{j} \in \mathbb{C} \backslash\{0\}$. With this, (3.1) reads

$$
\operatorname{Im}\left(\zeta_{j} \bar{\zeta}_{k}\right) L_{j}^{\mathbb{R}} \wedge L_{k}^{\mathbb{R}}=0
$$

implying either $\operatorname{Im}\left(\zeta_{j} \bar{\zeta}_{k}\right)=0$ or $L_{j}^{\mathbb{R}} \wedge L_{k}^{\mathbb{R}}=0$. We can relabel all the vector fields $L_{j}$ having the property that $L_{j}^{\mathbb{R}} \wedge L_{1}^{\mathbb{R}}=0$ so that they become $L_{1}, \ldots, L_{\nu_{1}}$. Next we relabel all the vector fields $L_{j}$ such that $L_{j}^{\mathbb{R}} \wedge L_{\nu_{1}+1}^{\mathbb{R}}=0$ so that they become $L_{\nu_{1}+1}, \ldots, L_{\nu_{2}}$, etc. We end up with a set of vector fields $X_{1}, \ldots, X_{r}(1 \leq r \leq m)$ with real constant coefficients such that $X_{\alpha} \wedge X_{\beta} \neq 0$ if $\alpha \neq \beta$ and such that to each $j \in[1, \ldots, m]$ there is $\alpha \in[1, \ldots, r]$ and $\zeta_{j}^{\prime} \in \mathbb{C} \backslash\{0\}$ such that $L_{j}=\zeta_{j}^{\prime} X_{\alpha}$. Moreover, if $L_{k}=\zeta_{k}^{\prime} X_{\beta}$ with $\alpha \neq \beta$, then $\operatorname{Im}\left(\zeta_{j}^{\prime} \bar{\zeta}_{k}^{\prime}\right)=0$, meaning that $\zeta_{j}^{\prime}=c_{j, k} \zeta_{k}^{\prime}$ for some $c_{j, k} \in \mathbb{R} \backslash\{0\}$. In particular, this implies $\zeta_{k}^{\prime}=c_{k, 1} \zeta_{j}^{\prime}$ for all $j=1, \ldots, \nu_{1}$ and all $k>\nu_{1}$. Moreover, when $r>1$, what we have just said implies $\zeta_{j}^{\prime}=c_{j, 1} \zeta_{1}^{\prime}$ for all $j \in\left[2, \ldots, \nu_{1}\right]$. We find ourselves in one of the following two situations:

(1) $r=1$; there is a single vector field $X$ with real constant coefficients such that $L_{j}=\zeta_{j} X$ for each $j=1, \ldots, m$, with $\zeta_{j} \in \mathbb{C} \backslash\{0\}$. In this case,

$$
L=\left(\sum_{j=1}^{m} \zeta_{j} x_{j}\right) X
$$

(2) $r>1$; there are numbers $\zeta \in \mathbb{C} \backslash\{0\}$ and $c_{\alpha, j} \in \mathbb{R} \backslash\{0\}$ such that

$$
L=\zeta \sum_{\alpha=1}^{r}\left(\sum_{j=\nu_{\alpha-1}+1}^{\nu_{\alpha}} c_{\alpha, j} x_{j}\right) X_{\alpha}
$$

When (3.3) holds Theorem 1 applies to $\zeta^{-1} L$ and therefore extends to $L$.

Since codim $\boldsymbol{V}(L)=m$, when (3.2) holds we have

$$
\sum_{k=1}^{m}\left(\operatorname{Re} \zeta_{k}\right) x_{k}=\sum_{k=1}^{m}\left(\operatorname{Im} \zeta_{k}\right) x_{k}=0 \Longrightarrow x_{1}=\cdots=x_{m}=0 .
$$

This is possible only if $m \leq 2$. Moreover, if $m=2$, it requires $\operatorname{Im}\left(\zeta_{1} \bar{\zeta}_{2}\right) \neq 0$; in turn the latter allows one to carry out a real linear change of the variables $x_{1}, x_{2}$ such that $L=\zeta\left(x_{1}+i x_{2}\right) X, 0 \neq \zeta \in \mathbb{C}$. If $m=1$, we must have $L=\zeta x_{1} X$. The only one of these two cases not covered by Theorem 1 (after division by $\zeta$ ) is the case of $L=\left(x_{1}+i x_{2}\right) X$, whose local solvability is a consequence of Lemma 2

If $L=\left(x_{1}+i x_{2}\right) X$ (and thus $m=2$ ), not every eigenvalue of $\mathfrak{m}\left(L^{\circ}\right)$ [see (1.4)] can be purely imaginary (and different from zero). Indeed, in an arbitrary basis of $\mathbb{R}^{2}$ we will have

$$
\mathfrak{m}\left(L^{\circ}\right)=\left(\begin{array}{cc}
a_{11} & a_{12} \\
i a_{11} & i a_{12}
\end{array}\right), a_{11}, a_{12} \in \mathbb{R},
$$

whose eigenvalues are $a_{11}+i a_{12}$ and 0 . The orbits of $L=\left(x_{1}+i x_{2}\right) X$ in $\mathbb{R}^{n} \backslash \boldsymbol{V}(L)$ are equal to the connected components of the intersections of $\mathbb{R}^{n} \backslash \boldsymbol{V}(L)$ with the 
orbits of $X$ in $\mathbb{R}^{n}$. The latter being straight lines, the closures of the orbits of $L$ in $\mathbb{R}^{n} \backslash \boldsymbol{V}(L)$ are not compact ( $X$ has real constant coefficients, not all equal to zero). We can state

Theorem 2. Let the vector field (1.1) have complex coefficients. If $L \wedge \bar{L}$ vanishes identically, then the properties (a), (b), (c) in Theorem 1 are equivalent.

\section{REFERENCES}

[Lojasiewicz, 1965] Lojasiewicz, S., Notes, Institut Hautes Études, Bures-sur-Yvette, 1965.

[Miwa, 1973] Miwa, T., On the existence of hyperfunction solutions of linear differential equations of the first order with degenerate real principal symbols, Proc. Japan Acad. 49 (1973), 88-93. MR0348236(50:734)

[Treves, 1992] Treves, F., Hypo-analytic Structures, Local Theory, Princeton University Press, Princeton, NJ, 1992. MR.1200459 (94e:35014)

[Treves, 2009] Treves, F., On planar vector fields with complex linear coefficients, to appear.

Department of Mathematics, Rutgers University, New Brunswick, New Jersey 089032101

E-mail address: treves.jeanfrancois@gmail.com 